\title{
Leptonic emission from microquasar jets: from radio to very high-energy gamma-rays
}

\author{
Valentí Bosch-Ramon ${ }^{1}$, Josep M. Paredes ${ }^{1}$ and Gustavo E. Romero ${ }^{2,3}$ \\ ${ }^{1}$ Departament d'Astronomia i Meteorologia, Universitat de Barcelona, Av. Diagonal 647, \\ E-08028 Barcelona, Catalonia (Spain) \\ email: vbosch@am.ub.es \\ ${ }^{2}$ Instituto Argentino de Radioastronomía, C.C.5, (1894) Villa Elisa, Buenos Aires (Argentina) \\ ${ }^{3}$ Facultad de Ciencias Astronómicas y Geofísicas, UNLP, Paseo del Bosque, 1900 La Plata \\ (Argentina)
}

\begin{abstract}
Microquasars are sources of very high-energy gamma-rays and, very probably, highenergy gamma-ray emitters. We propose a model for a jet that can allow to give accurate observational predictions for jet emission at different energies and provide with physical information of the object using multiwavelength data.
\end{abstract}

Keywords. X-rays: binaries, gamma rays: theory, gamma rays: observations

Microquasars are X-ray binaries with relativistic jets whose emission extends from radio to gamma-rays. In particular, LS 5039, discovered by Paredes et al. (2000), turned out to be the first likely high-energy gamma-ray microquasar due to its possible association with the EGRET source 3EG J1824-1514 (Paredes et al. 2000). Further theoretical studies furnished with more reliability this association (Bosch-Ramon \& Paredes 2004), which could be extended to other EGRET sources (Kaufman Bernadó et al. 2002, Romero et al. 2003, Bosch-Ramon et al. 2005a). Very recently, Aharonian et al. (2005) have published the detection of the microquasar LS 5039 at TeV energies, leaving no doubt about the gamma-ray emitting nature of these objects. The aim of the present work is to show that from the theoretical point of view microquasar jets can be sources of emission with energies covering the whole spectral band.

In our model, the jet is modelled as dynamically dominated by cold protons and radiatively dominated by relativistic leptons. The characteristics of the orbit and the companion star constrain the way in which the accretion takes place. This has been taken into accout for a consistent orbital variability treatment. The magnetic field energy density and the non-thermal particle maximum energy values along the jet depend on the cold matter energy density and the particle acceleration/energy loss balance, respectively, and the amount of relativistic particles within the jet is restricted by the efficiency of the shock to transfer energy to them. The model takes into account the external and internal photon and matter fields, which interact with relativistic particles in the magnetized jet, producing emission from radio to very high energies. Concerning the the physical parameters of the model, we have adopted the typical values for a massive X-ray binary system and the standard ones in jet and acceleration theory (OB star, semi-major axis of about $0.1 \mathrm{AU}$, strong stellar wind, jet matter/accretion matter rate ratio of about 0.1 , magnetic field of about a $10 \%$ of the equipartition one, acceleration efficiencies $10^{-3}$ $10^{-1} \mathrm{qBc}$ ). For further details concerning the model, see Bosch-Ramon et al. (2005b).

This model has been applied to LS 5039. In Fig. 1, we show the computed SED, and the flux and photon index evolution along the orbit for this source. The model underpredicts the fluxes in the radio and $\mathrm{TeV}$ band. Although the difference is less than one order 
of magnitude, it could be interpreted as a hint that more intense high energy processes (particle acceleration and emission) are taking place already outside the binary system, since radiation is emitted mainly within the $100 \mathrm{GeV}$-photon absorption region close to the companion star. This could also lead to higher fluxes in radio, due to this radiation is optically thin when emitted outside the binary system. Concerning the variability of the source along the orbit, we have used a particular accretion model for this source consisting in a slow equatorial wind from a fast rotating stellar companion (Casares et al. 2005), altogether with a stream of matter ejected during the periastron passage and reaching the compact object at phase 0.8-0.9, when the X-ray (Bosch-Ramon et al. 2005c) and the TeV peaks (Aharonian et al. 2005, Casares et al. 2005) are observed. For more details concerning the application of the model to LS 5039, see the work of Paredes et al. (2005). It is worth noting that the total jet kinetic power required for producing a SED like the one plotted in Fig. 1 is few times $10^{36} \mathrm{erg} / \mathrm{s}$. Therefore, the very likely nature of microquasars and, in particular, LS 5039 as $\mathrm{GeV}-\mathrm{TeV}$ emitters appears well founded from theoretical grounds.
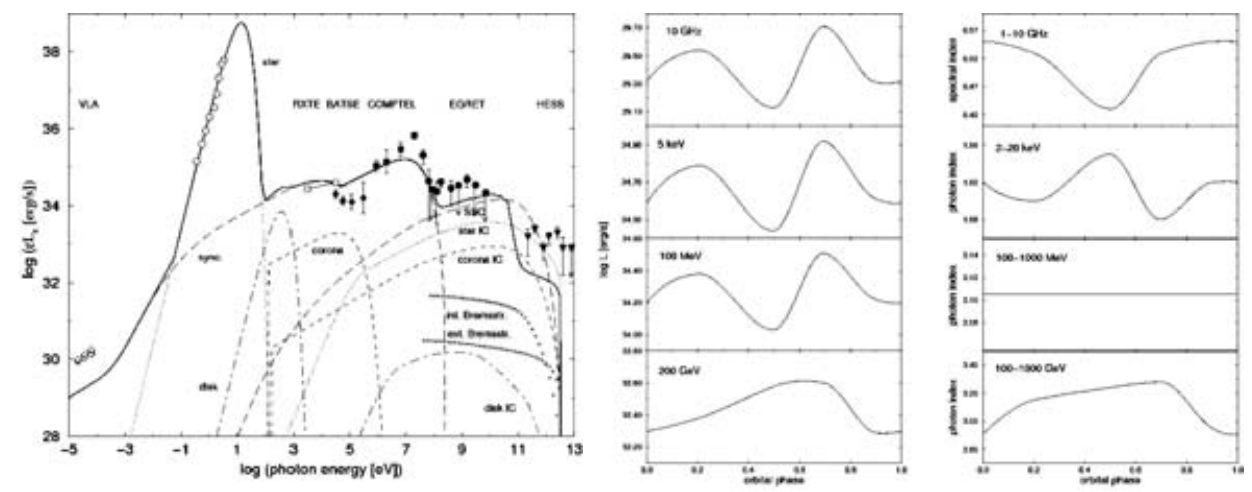

Figure 1. a) Computed SED for LS 5039 and b) its flux and photon index evolution at different energy bands.

\section{Acknowledgements}

V.B-R. and J.M.P. acknowledge partial support by DGI of the spanish Ministerio de Educación y Ciencia under grant AYA2004-07171-C02-01, as well as additional support from the European Regional Development Fund (ERDF/FEDER). During this work, V.B-R. has been supported by the DGI of the spanish Ministerio de Educación y Ciencia under the fellowship BES-2002-2699. G.E.R is supported by the Argentine Agencies CONICET and ANPCyT (PICT 03-13291).

\section{References}

Aharonian, F. et al. 2005, Science 309, 746

Bosch-Ramon, V. \& Paredes, J. M. 2004, A\&A 417, 1075

Bosch-Ramon, V. Romero, G. E. \& Paredes, J. M. 2005a, A\&A 429, 267

Bosch-Ramon, V. Romero, G. E. \& Paredes, J. M. 2005b, A\&AA, submitted

Bosch-Ramon, V., Paredes, J. M., Ribó, M., Miller, J. M., Reig, P., Martí, J. 2005c, ApJ 628, 1 Casares, J., Ribó, M., Ribas, I., Paredes, J. M., Martí, J., Herrero, A. 2005, MNRAS, submitted Kaufman Bernad, M., Romero, G. E. \& Mirabel, I. F. 2002, A\& A 385, 10

Paredes, J. M., Mart, J., Ribó, M. \& Massi, M. 2000, Science 288, 2340

Paredes, J. M., Bosch-Ramon, V. \& Romero, G. E. 2005, A\&A, submitted

Romero, G. E., Torres, D. F., Kaufman Bernadó, M., Mirabel, I. F. 2003, A\&\&A 410, 1 